\title{
Errata for "A robustness approach to international sourcing" by G.J. Gutierrez and P. Kouvelis, Annals of Operations Research 59 (1995) 165-193
}

\section{Genaro J. Gutierrez • Panagiotis Kouvelis • Lawrence V. Snyder}

Published online: 29 March 2008

(C) Springer Science+Business Media, LLC 2008

The second part of Step 5 of the algorithm on page 184 reads:

If $\left[\left|L_{R}\right|>N\right]$

then \{Drop the $\left|L_{R}\right|-N$ solutions $k$ with the largest $R^{k}$, and also drop all their corresponding nodes in all trees,

$$
\text { Set } p=\max _{k \in L_{R}}\left\{R^{k}\right\}
$$

Go to Step 1.

It should read as follows:

If $\left[\left|L_{R}\right|>N\right]$

then $\left\{\right.$ Drop the $\left|L_{R}\right|-N$ solutions $k$ with the largest $R^{k}$, set $p=\max _{k \in L_{R}}\left\{R^{k}\right\}$, and \} drop from all trees the nodes $k$ with $\frac{\underline{Z}_{s}^{k}-Z_{s}\left(Y_{s}^{*}\right)}{Z_{s}\left(Y_{s}^{*}\right)}>p$ for any $s \in S$.

Go to Step 1.

The need for this correction was pointed out to us by L.V. Snyder. 